\title{
Amoebic forms of Blastocystis spp. - evidence for a pathogenic role
}

\author{
Arutchelvan Rajamanikam and Suresh Kumar Govind*
}

\begin{abstract}
Background: Blastocystis spp. are one of the most prevalent parasites isolated from patients suffering from diarrhea, flatulence, constipation and vomiting. It's pathogenicity and pathophysiology remains controversial to date. Protease activity and amoebic forms have been reported previously in symptomatic isolates but there has been no conclusive evidence provided to correlate the protease activity and any specific life cycle stage of the parasite thus far.

Methods: Symptomatic isolates with amoebic form were tested for protease activity and compared with symptomatic and asymptomatic isolates without amoebic form for 10 days culture period.

Results: The present study demonstrates an elevated protease activity in cultures having a higher percentage of amoebic forms seen in symptomatic isolates. The growth curve demonstrated a significantly $(p<0.05)$ higher average number of parasite counts in asymptomatic compared to symptomatic isolates. Symptomatic isolates showed amoebic forms with percentages ranging from $5 \%$ to $17 \%$. Elevated protease activity was demonstrated in isolates that had higher percentages of amoebic forms with intense bands at higher molecular weight proteases $(60-100 \mathrm{kDa})$. As days of culture proceeded, the protease quantification also showed a steady increase.
\end{abstract}

Conclusion: This study elucidates a correlation between protease activity and percentage of amoebic forms. The finding implies that these forms could play a role in exacerbation of intestinal symptoms during Blastocystis spp. infection.

Keywords: Blastocystis spp, Protease activity, Amoebic form, Gastrointestinal

\section{Background}

Blastocystis spp., a controversial anaerobic parasite, has increasingly gained a reputation for being implicated in causing flatulence, diarrhea, constipation, vomiting and skin rash [1-3]. The mechanism of pathogenicity still remains unclear. The prevalence in humans is reported to be between $0.5 \%$ [4] to $60 \%$ [5] and $40-60 \%$ in animals $[2,6]$.

Genotypic variability has been reported to play an influential role in the pathogenicity of Blastocystis [7]. This is evidenced by studies showing Blastocystis subtype 3 being the most common subtype isolated from patients with various gastrointestinal (GI) disorders. Despite a few prevalence studies that have been carried out in different countries, which showed subtype 3 followed by subtype 1 to be the highest [7-10], its pathogenic role has not been clearly defined. The question of whether

\footnotetext{
*Correspondence: suresh@um.edu.my

Department of Parasitology, University of Malaya, Kuala Lumpur 50603, Malaysia
}

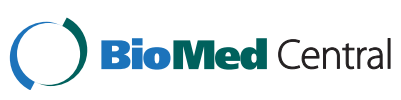

(c) 2013 Rajamanikam and Govind; licensee BioMed Central Ltd. This is an Open Access article distributed under the terms of the Creative Commons Attribution License (http://creativecommons.org/licenses/by/2.0), which permits unrestricted use, distribution, and reproduction in any medium, provided the original work is properly cited. The Creative Commons Public Domain Dedication waiver (http://creativecommons.org/publicdomain/zero/1.0/) applies to the data made available in this article, unless otherwise stated. there are other factors influencing the parasite in its ability to be opportunistic in immunocompetent and immunocompromised patients $[2,11]$ has not yet been clearly answered.

There are also reports that demonstrate the lack of correlation between the symptomatic and asymptomatic group in terms of genotype distribution [12-14] and whether this is related to factors such as age of host and genetics is still uncertain [7]. Amoebic forms, one of the life cycle stages of the parasite, have been reported to be present in higher percentages in in vitro cultures of symptomatic isolates compared to asymptomatic isolates $[15,16]$. Previously, amoebic forms were also observed in colonoscopic lavage and patients with acute diarrheal syndrome $[17,18]$.

Proteases from Blastocystis spp. have been proven to be one of the important candidates that contribute to the pathogenicity of this protozoan parasite $[19,20]$. Proteases from Blastocystis spp. have been shown to 
be able to degrade immunoglobin A (Ig A) giving rise to the parasite's ability to have greater virulence and colonization through the elicitation and alteration of immunological response as well as the disruption of barrier function [21-23].

To date there have been no studies to correlate the presence of amoebic forms in in vitro culture and protease activity of Blastocystis spp. The present study attempts to elucidate if there is a correlation between the percentage of amoebic forms in cultures isolated from asymptomatic and symptomatic patients to protease activity.

\section{Methods}

\section{Source of Blastocystis spp.}

Parasites were obtained from random stool sample collection in a survey carried out in a particular rural area in Selangor, Malaysia. A total of 5 isolates from symptomatic (S1-S5) and asymptomatic (A1-A5) patients were continuously cultured in Jones' medium. The symptomatic isolates originated from patients showing symptoms such as flatulence, abdominal pain, diarrhea and constipation. This information was obtained using a questionnaire. Samples with Blastocystis spp. were selected through direct fecal screening and cyst concentration technique to select the samples with only Blastocystis spp. as the sole symptom causative agent.

\section{Culture and purification of Blastocystis spp.}

All isolates were inoculated in Jones' medium supplemented with $10 \%$ horse serum as described previously by Suresh et al. [24]. Cultures were incubated at a constant temperature of $37^{\circ} \mathrm{C}$ before sub-culturing into fresh medium once every 3 days. Subsequently, the cells were purified from bacterial-contaminated culture using density gradient centrifugation. The cells were pooled into one tube and washed twice with phosphate buffered saline (PBS) for 5 minutes at $500 \mathrm{~g}$. Five milliliters of the cell suspension was then layered carefully onto $6 \mathrm{ml}$ of FicollPaque without agitation. It was then spun for 20 mins at 700 g. Blastocystis spp. cells with minimal bacterial contaminants found above the thick layer of yellowish white clump was then gently isolated and washed with PBS. The pellet was stored at $-20^{\circ} \mathrm{C}$ until further use.
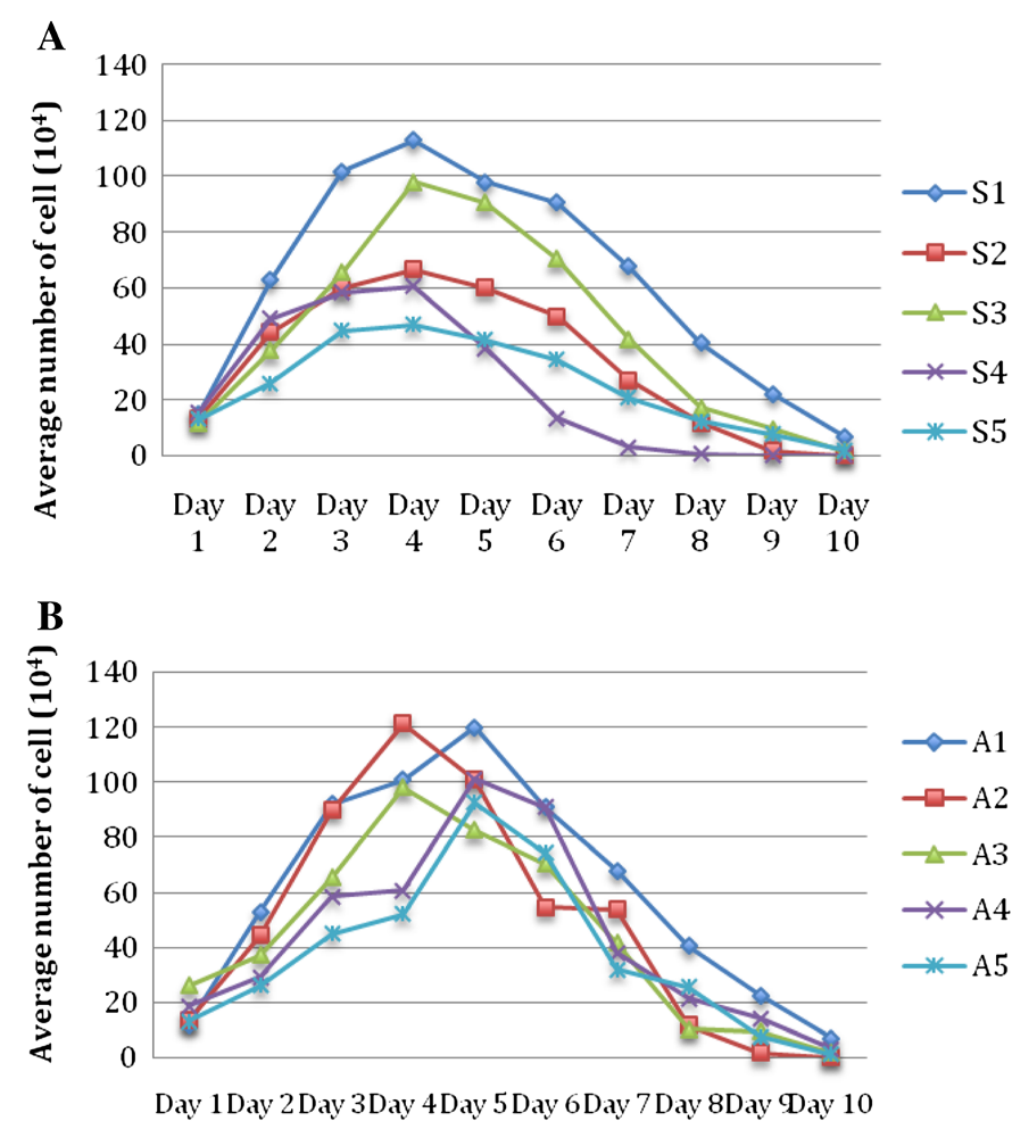

Figure 1 Growth profile of Blastocystis spp. isolated from symptomatic (A) and asymptomatic (B) patients. A comparison on the average number of cells is exhibited. 


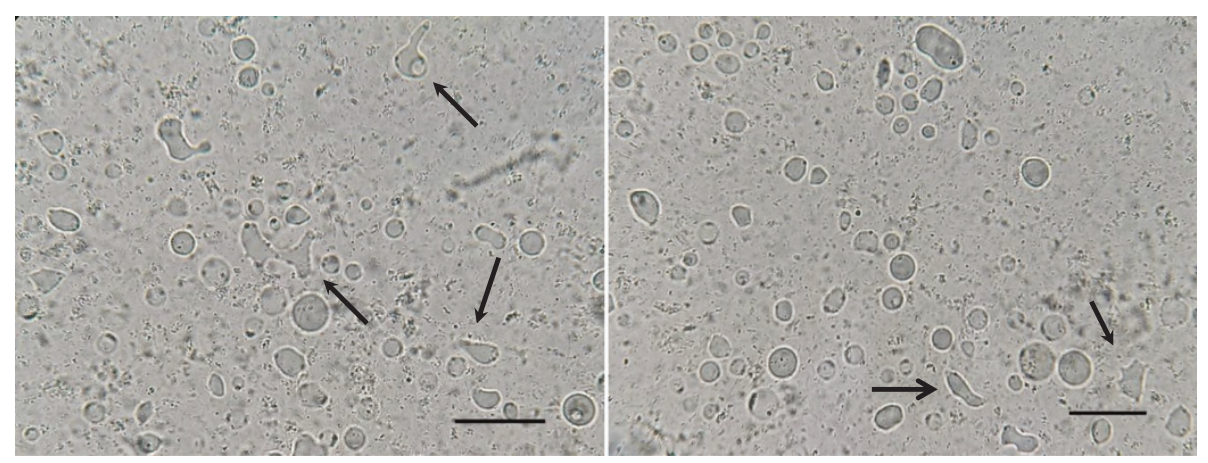

Figure 2 Amoebic forms found in in vitro culture of symptomatic (S2) isolates on day 5. Amoebic forms with protruding cytoplasm are indicated with arrow. Bar $=10 \mu \mathrm{m}$.

\section{Morphological study to elucidate morphological forms in culture}

Isolates, maintained for a few weeks and showing consistent growth were used subsequently for the elucidation of morphological forms in culture. $10^{5}$ cells in vacuolar form were counted and inoculated into culture tubes containing $1 \mathrm{ml}$ of Jones' medium with $10 \%$ horse serum and maintained to determine the growth profile at $37^{\circ} \mathrm{C} .10$ of culture suspension containing the parasites was mixed with an equal volume of Trypan blue to assess cell viability. The isolates were screened under a light microscope with 40X magnification everyday for 10 consecutive days and the percentages of amoeboid cells were observed by counting the number of amoeboid forms in a random count of 100 cells [15]. Parasites from all culture tubes on day 5 were collected, purified and the respective pellets were then stored at $-20^{\circ} \mathrm{C}$. In a separate experiment, cell lysates of Blastocystis spp. isolates from all culture tubes were obtained at different periods of incubation (Day 1 ,
3, 5 and 7), which were then subjected to the GelatinSDS-PAGE and azocasein assays for the detection of protease activity using a standardized protein concentration of $0.1 \mathrm{mg} / \mathrm{ml}$.

\section{Gelatin-SDS-PAGE for the detection of protease activity}

Purified isolates were washed twice with $0.85 \% \mathrm{NaCl}$. The respective pellets were then re-suspended with 200 of lysis buffer containing $4.3 \mathrm{~g}$ of sucrose and $100 \mathrm{mM}$ of $\mathrm{NaCl}$ in $1 \%$ triton-X and kept overnight at $4^{\circ} \mathrm{C}$. Protein concentration was then estimated using the Bio-Rad Microassay method (Bio-Rad). Twelve percent of resolving and $5 \%$ of stacking gel were prepared. Cell lysates with standardized protein concentration were then electrophoresed as described previously [20]. The gel was then subjected to re-naturation and incubation to observe the protease activity. Subsequently, the gel was stained with 0.12\% (w/v) Coomassie Brilliant Blue R-250 for 1 hour. The protease bands appear as colorless bands with a dark blue background.

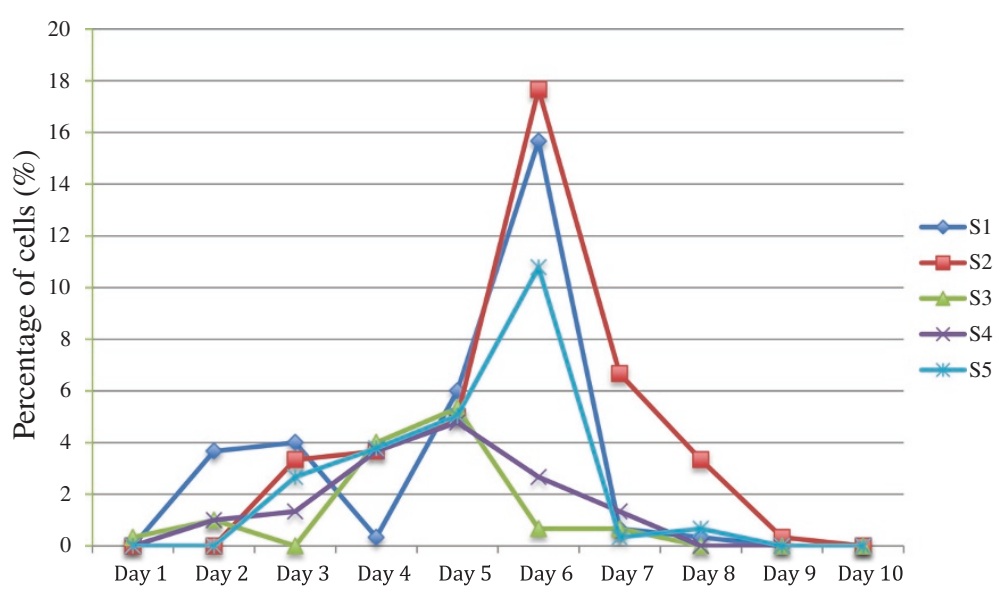

Figure 3 Percentage of amoeboid forms in symptomatic isolates over a 10 days growth period. All isolates were inoculated in Jones medium supplemented with $10 \%$ horse serum at 37C. Note: Initial culture volume was $10^{5}$ cells $/ \mathrm{ml}$. 


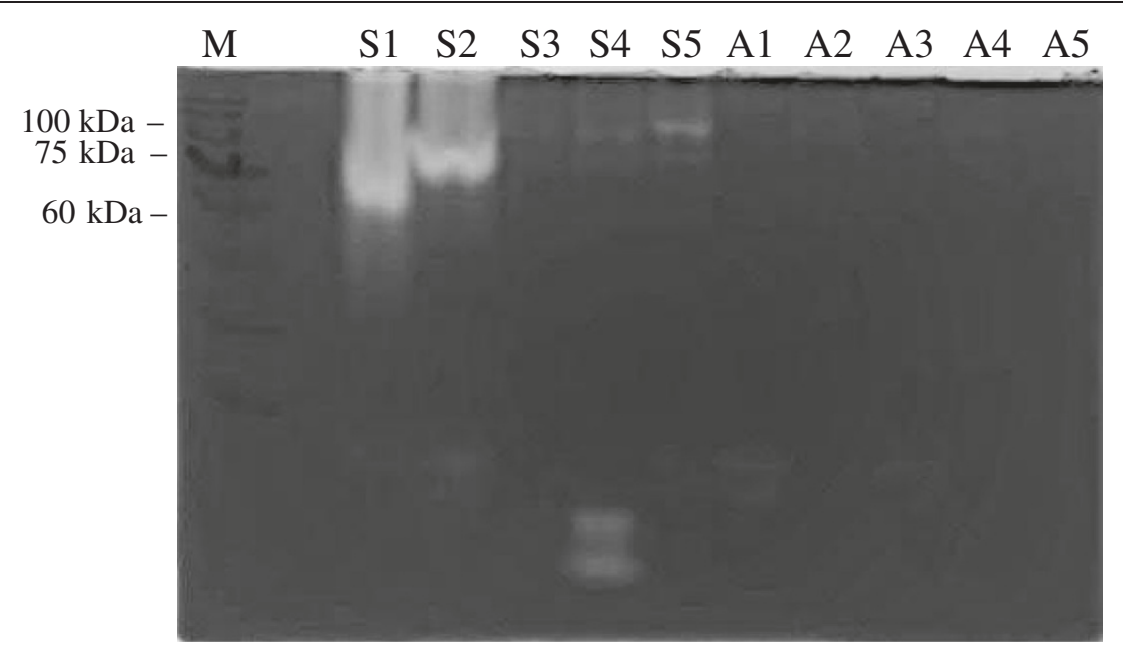

Figure 4 Protease profile from the cell lysates of symptomatic isolates (S1-S5) and asymptomatic isolates (A1-A5) of Blastocystis spp. The concentration of the cell lysate was standardized $(0.1 \mathrm{mg} / \mathrm{ml})$ and the lysates of each isolate was harvested on day 5 of incubation at $37 \mathrm{C}$.

\section{Azocasein assay for colorimetric quantification of protease activity}

Parasites were purified as described previously. The cells were lysed through a series of 15 cycles of freeze and thaw. The tubes containing the respective pellets were stored at $4^{\circ} \mathrm{C}$ overnight. The contents of each tube were centrifuged to sediment the debris and the protein content was estimated using the Bio-Rad Microassay method (Bio-Rad). The concentration of each sample was standardized before the assay. The cell lysates were initially incubated for 10 minutes with the supplementation of DTT $(2 \mathrm{mM})$ (Sigma-Aldrich) at $37^{\circ} \mathrm{C}$. This activated the parasitic proteases. $5 \mathrm{mg} / \mathrm{ml}$ of Azocasein was prepared in PBS at $\mathrm{pH}$ 7.4. One hundred microliters of lysate was mixed with 100 of pre-heated $\left(37^{\circ} \mathrm{C}\right)$ azocasein solution and left to incubate at $37^{\circ} \mathrm{C}$ for 1 hour. The reaction was stopped by the addition of 300 of ice-cold Trichloroacetic acid and the mixture was then left on ice for 30 minutes. The tubes were then centrifuged at $8000 \mathrm{~g}$ to remove the undigested azocasein and the supernant was subsequently added to 500 of $\mathrm{NaCl}(500 \mathrm{mM})$. The clear orange solution that was produced was measured at $440 \mathrm{~nm}$ absorbance. Trypsin and inactivated lysate were used as positive and negative controls respectively. This experiment was carried out in triplicate.

\section{Statistical analysis}

All statistical analysis was carried out using IBM $^{\odot}$ SPSS $^{\odot}$ Statistics Version 21. Independent Students t-test was used to assess the difference in the number of cells and

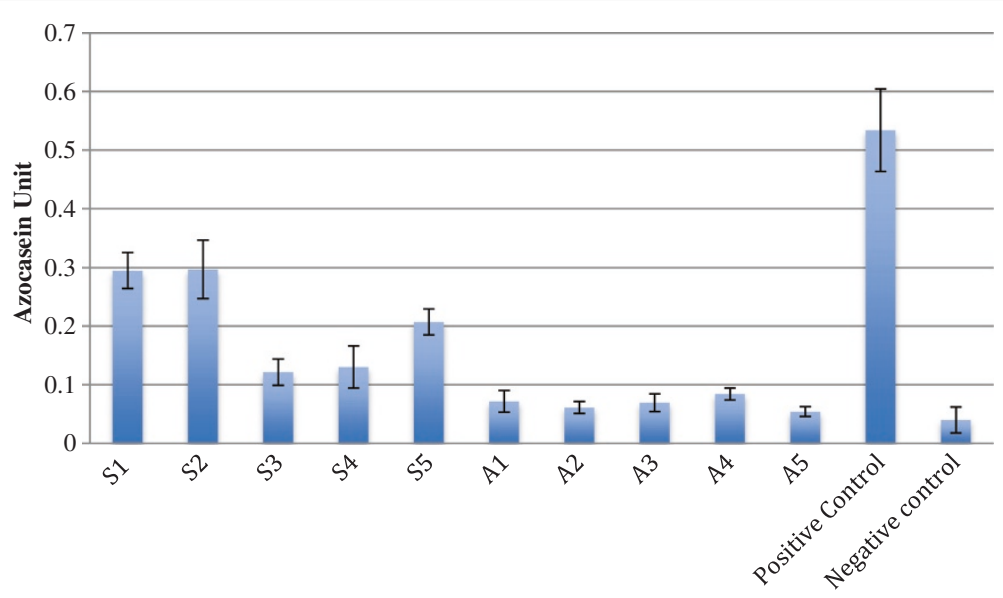

Figure 5 Protease activity of symptomatic (S1-S5) and asymptomatic (A1-A5) isolates of Blastocystis spp. on day 5 of incubation. The concentration of cell lysate was standardized to $0.1 \mathrm{mg} / \mathrm{ml}$. Values are obtained from an average of three independent experiments with triplicates in each samples and standard deviations are indicated as error bars. 
protease activity between symptomatic isolates with amoebic forms, symptomatic isolates without amoebic forms and asymptomatic isolates. Pearson Correlation Test was used to assess the correlation between protease activity and percentage of amoebic forms. A value of $\mathrm{p}<0.05$ is considered statistically significant.

\section{Results}

Growth profile studies were carried out in all five symptomatic and asymptomatic isolates respectively over a 10 day period. The growth profile of both symptomatic and asymptomatic isolates for a 10 day culture period showed significantly $(\mathrm{p}=0.008)$ higher peak parasite

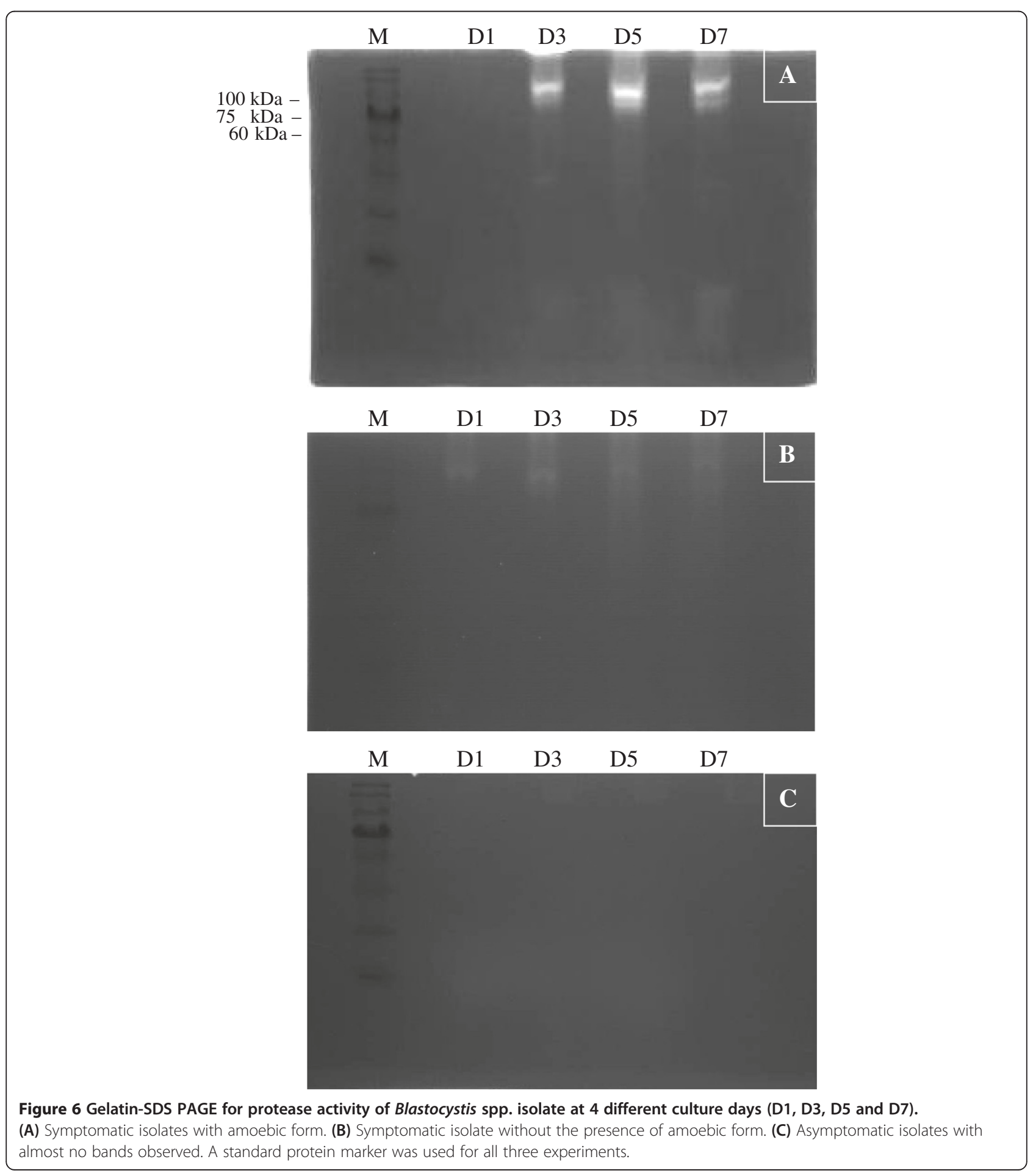


counts in asymptomatic isolates on day 5 to day 7 (Figure 1). All symptomatic isolates (S1 to S5) showed amoebic forms over the 10 day culture period with a size range between $2.9-8.0 \mu \mathrm{m}$ (Figure 2). Amoebic forms were first observed on day 3 with cells showing multiple extended irregular shaped cytoplasm and a prominent nucleus. These forms persisted until day 7 (S1, S3 and S5) to day 8 (S2 and $\mathrm{S} 4$ ) with the percentage ranging from $5 \%$ to $17 \%$ (Figure 3). Higher percentages of amoebic form were observed in S2 (18\%) followed by S1 and S5, which was $16 \%$ and $11 \%$ respectively. S3 and S4 showed the least number of amoebic forms. Asymptomatic isolates showed no amoebic forms in culture but had a higher parasite count with forms being mostly vacuolar followed by granular (data not shown).

Protease activity using the azocasein assay and gelatinSDS-PAGE demonstrated higher activity in symptomatic isolates with the highest observed in S2 followed by S1, S5, S4 and S3. Asymptomatic isolates demonstrated a consistently lower activity of protease in all five isolates.

Elevated protease activity was demonstrated in isolates that exhibited higher percentages of amoebic forms with intense bands (Figure 4). The variation in the intensity of the band was observed only with higher molecular weight proteases $(60-100 \mathrm{kDa})$. Protease banding patterns in current protease zymography showed a similar outcome as in the azocasein assay (Figure 5). The bands were more intense in S1, and S2 whereas the bands in S3, S4 and S5 showed less intense bands. However, only high molecular weight protease bands were observed to correlate with the percentage of amoebic forms seen in culture. Lower molecular weight protease did not show much correlation and an intense band was seen only in isolate $\mathrm{S} 4$ at $28-17 \mathrm{kDa}$.

As days of culture preceded the protease quantification (Figure 6) also showed a steady increase in the band intensity until day 5 with a slight decrease observed only on day 7. Apparently, there was no obvious variation in the band intensity observed in symptomatic isolates without amoebic forms. A similar outcome was observed for asymptomatic isolates with only very faint bands spotted for all 4 different incubation days. A similar lysate concentration of symptomatic isolates with amoebic forms, symptomatic isolates without amoebic forms and asymptomatic isolates were used for the azocasein assay Figure 7. The results showed almost equal increases in protease activity on days 1, 3, 5 and 7 of the culture period. However, protease activity of symptomatic isolates with amoebic forms was significantly higher than the rest.

Symptomatic isolates with amoebic forms showed significantly higher activity of protease compared to symptomatic isolates without amoebic forms $(p=0.014)$ and asymptomatic isolates $(\mathrm{p}=0.003)$. Furthermore, a strong correlation between the percentage of amoebic forms and average protease activity was observed (Table 1 ).

\section{Discussion and conclusions}

This study is the first to report a correlation between the presence of amoebic forms and protease activity of Blastocystis spp. Proteases have previously been shown to be involved as one of the pathogenic factors of Entamoeba histolytica, Trichomonas vaginalis and Acanthamoeba sp. [25-27]. In a previous study on Blastocystis spp., subtype 3 of symptomatic isolates were shown to frequently posses protease activity especially at $32 \mathrm{kDa}$, which was postulated to be the virulent factor [20].

Cysteine-type activity of protease was observed in Blastocystis spp. isolated from patients with gastrointestinal symptoms [19] with its effect on intestinal cells and gut functions [28]. Proteases from Blastocystis spp. have also been shown to cleave intestinal IgA and elicit

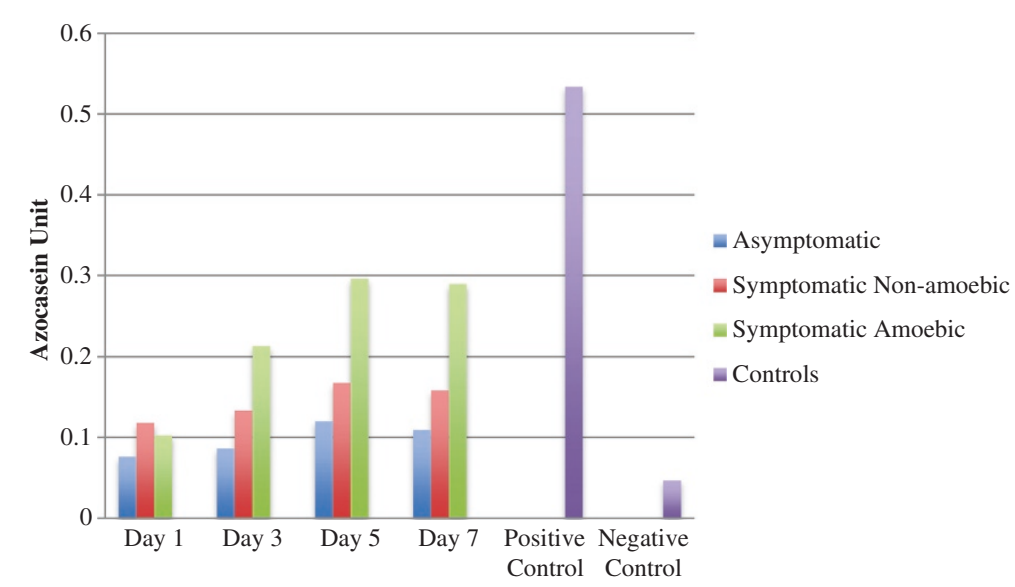

Figure 7 Protease activity at $\mathbf{4}$ different points of incubation. Trypsin was used as positive control and heat-inactivated lysate was used as negative control. Values were obtained from an average of three independent experiments with triplicates in each sample and standard deviations are indicated as error bars. 
Table 1 Pearson's correlation of average protease activity and average amoebic form count

\begin{tabular}{ccc}
\hline & p & $\begin{array}{c}\text { Correlation } \\
\text { coefficient(r) }\end{array}$ \\
\hline Protease activity and amoebic cell count & 0.036 & 0.964
\end{tabular}

Very high correlation is observed with significance level at $\mathrm{p}<0.05$.

inflammatory cytokines [21,22]. However, these reports did not attempt to provide credible evidence on the life cycle stage of Blastocystis spp. responsible for protease activity.

The relationship of proteases to pathogenicity can be better illustrated with the example of Entamoeba histolytica where its membrane-associated serine proteases, with a molecular weight $60 \mathrm{kDa}$, were shown to be responsible for the degradation lysosamal proteins and tight junctions in target cells. These proteases were found in low levels in the non-pathogenic strain, Entamoeba dispar [29]. Proteases from Trypanosoma cruzi [30] and Acanthamoeba [31] have been shown to play a role in immune evasion during Chagas disease and be responsible for extracellular membrane degradation during granulomatous encephalitis respectively.

Amoebic forms were shown to be a life cycle stage of Blastocystis spp. [32] as well as being seen in cultures isolated from symptomatic patients [16,32,33]. Amoebic forms have also been reported in stool specimens of patients with diarrhea [34]. In the present study we also saw amoebic forms only in symptomatic isolates. Amoebic forms have been shown to be responsible for the engulfment of bacteria [32] which could then survive and replicate within lysosomes or phagosomes [35]. Whether bacteria contribute to the pathogenic role of the parasite still remains in question.

The present study demonstrated intense protease activity seen on day 5 in all symptomatic isolates, which correspondingly co-related to the intense bands of 60$100 \mathrm{kDa}$ seen in the same day having the higher percentage of amoebic forms observed in all isolates. The presence of lower molecular weight $(17-28 \mathrm{kDa})$ protease in isolates $\mathrm{S} 2$ and $\mathrm{S} 4$ as well as in asymptomatic isolates (A1 and A3) elicits a question on the significance of this band, which remains unclear although lower molecular weight bands in other studies have been suggested to be a virulence factor $[19,20]$.

Moreover, our study suggests a steady increase in protease activity during the incubation corresponding to an increase in amoebic forms and a slight decrease on day 7 , which relates to the drop in amoebic forms. A previous study reported that the fluctuation of cell size and protease activity appears to have a strong correlation when examined over a 96 hour incubation period [23]. This study contradicts our observation in that other than the percentage of amoebic forms, size of cell showed no influence on the protease activity. The present study involved a painstaking and tedious process to screen large samples of stools to obtain Blastocystis infected stool samples. We limited the study to a sample size of 5 as we had to obtain positive stool samples from symptomatic and asymptomatic patients, given that other published papers relating protease activity and Blastocystis spp. as well as other parasites have been carried out on a smaller sample size yet demonstrated, as in our study, significant and consistent results $[19,21,27,36]$. Similar studies should be carried out with larger sample sizes with isolates that have undergone genotypic characterization to statistically concrete our postulation and to completely understand the variation of protease activity in different life cycle stages of Blastocystis spp.

Our axenization attempts purified the parasite with minimal bacterial concentration. In the present study,

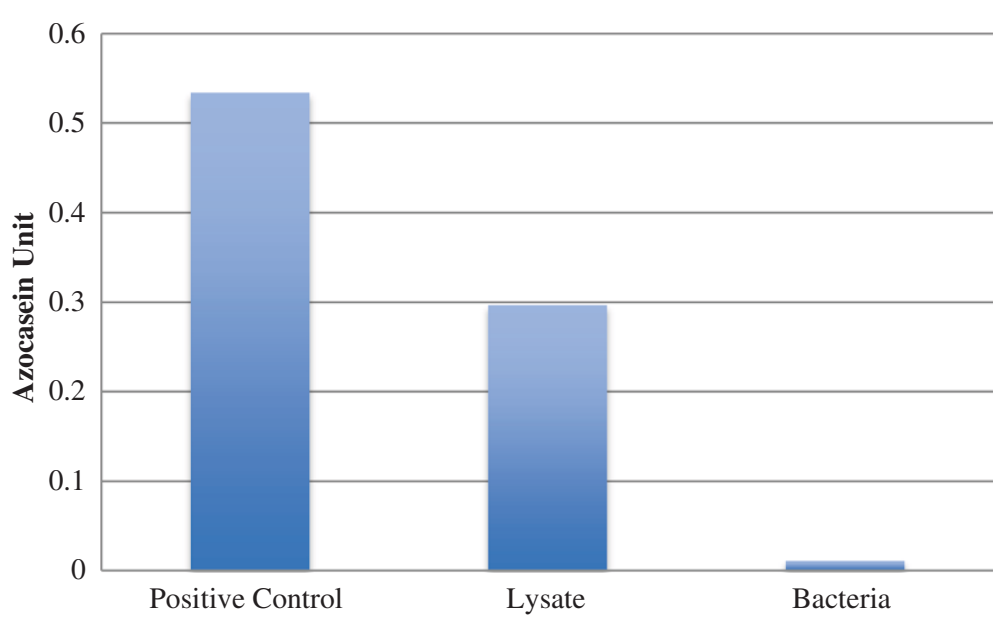

Figure 8 Assessment of bacterial contamination in cell lysates of Blastocystis spp. used in this study. Graph shows the protease activity of bacterial contaminant in cell lysate. 
lysates were extracted from purified xenic culture isolates. This was thought to mimic an environment similar to the natural intestinal microflora [15]. We have also shown that cell lysates with bacterial proteins had minimal effect since the bacterial protein demonstrated minimal protease activity (Figure 8 ).

The peak parasite count (parasite count between day 5 - 7) was used as one of the parameters in a previous study to differentiate the phenotypic characteristics of Blastocystis spp. It has been reported that asymptomatic isolates grew faster and the average total number of cells reached up to 7 times more than symptomatic isolate. The generation time was twice as high in asymptomatic isolates [37]. The present study concurred with these findings in that the asymptomatic isolates showed higher parasite peak counts than symptomatic ones (Figure 2). It is therefore evident that protease activity did not arise despite the higher parasite count consisting of mostly vacuolar and granular forms seen in asymptomatic isolates but from the larger percentage of amoebic forms seen in symptomatic isolates.

The amoebic form of Blastocystis spp. has been postulated previously to posses a sticky surface, which enhances the adherence to the intestinal epithelial cell lining [16]. This adherence could facilitate the release of proteases from amoebic forms to degrade extracellular membrane similar to that as seen in Entamoeba histolytica [38]. Higher occurrence of Blastocystis spp. has been seen in cancer patients undergoing chemotherapy [39]. Exacerbation of symptoms in such situations could be attributed to vacuolar stages reverting to amoebic forms, which in turn could be causing the related symptoms due to the release of proteases.

In conclusion, this is the first study to provide evidence co-relating amoebic forms of Blastocystis spp. and protease activity, which suggests a strong pathophysiological role that amoebic forms can play in exacerbating symptoms in patients infected with Blastocystis spp.

\section{Ethical approval}

This study was approved by the Medical Ethics Committee of the University Malaya Medical Centre (UMMC) (Kuala Lumpur, Malaysia) according to the Declaration of Helsinki approved this study.

\section{Competing interest}

Both authors declared that they have no competing interest.

\section{Authors' contribution}

$A R$ and SKG were involved in the intellectual planning of the experiment: SKG designed the study; AR carried out the experimental work; AR and SKG analysed the results and wrote the paper. Both authors read and approved the final manuscript.

\section{Acknowledgements}

We would like to extend our gratitude and heartfelt thanks to our lab collogues and all the staff in the Department of Parasitology, University of Malaya.

\section{Funding}

This study was entirely supported by Postgraduate Research Grant from University of Malaya (PG108-2012B) and High Impact Research Grant (HIR) (UM.C/625/1/HIR/031)

Received: 3 October 2013 Accepted: 3 October 2013

Published: 11 October 2013

References

1. Hussainqadri SM, Al-Okaili GA, Al-Dayel F: Clinical significance of Blastocystis hominis. J Clin Microbiol 1989, 27:2407-2409.

2. Tan KSW: New insights on classification, identification, and clinical relevance of Blastocystis spp. Clin Microbiol Rev 2008, 21:639-665.

3. Stenzel DJ, Boreham PFL: Blastocystis hominis revisited. Clin Microbiol Rev 1996, 9:563-584

4. Horiki N, Maruyama M, Fujita Y, Yonekura T, Minato S, Kaneda Y: Epidemiologic survey of Blastocystis hominis infection in Japan. Am J Trop Med Hyg 1997, 56:370-374.

5. Pegelow K, Gross R, Pietrzik K, Lukito W, Richards A, Fryauff DJ: Parasitological and nutritional situation of school children in the Sukaraja district, West Java. Indonesia. Southeast Asian J Trop Med Public Health 1997, 28:173-190.

6. Tan TC, Tan PC, Sharma R, Sugnaseelan S, Suresh KG: Genetic diversity of caprine Blastocystis from Peninsular Malaysia. Parasitol Res 2012, 112:85-89.

7. Souppart L, Sanciu G, Cian A, Wawrzyniak I, Delbac F, Capron M, Dei-Cas E, Boorom K, Delhaes L, Viscogliosi E: Molecular epidemiology of human Blastocystis isolates in France. Parasitol Res 2009, 105:413-421.

8. Dogruman-Al F, Dagci H, Yoshikawa H, Kurt Ö, Demirel M: A possible link between subtype 2 and asymptomatic infections of Blastocystis hominis. Parasitol Res 2008, 103:685-689.

9. Li LH, Zhang XP, Lv S, Zhang L, Yoshikawa H, Wu Z, Steinmann P, Utzinger J Tong XM, Chen SH, Zhou XN: Cross-sectional surveys and subtype classification of human Blastocystis isolates from four epidemiological settings in China. Parasitol Res 2007, 102:83-90.

10. Kaneda Y, Horiki N, Cheng X-J, Fujita Y, Maruyama M, Tachibana H: Ribodemes of Blastocystis hominis isolated in japan. Am J Trop Med Hyg 2001, 65:393-396.

11. Moosavi A, Haghighi A, Mojarad EN, Zayeri F, Alebouyeh M, Khazan H, Kazemi B, Zali MR: Genetic variability of Blastocystis sp. isolated from symptomatic and asymptomatic individuals in Iran. Parasitol Res 2012, 111:2311-2315.

12. Böhm-Gloning B, Knobloch J, Walderich B: Five subgroups of Blastocystis hominis from symptomatic and asymptomatic patients revealed by restriction site analysis of PCR-amplified 16S-like rDNA. Trop Med Int Health 1997, 2:771-778.

13. Yoshikawa $H$, Abe $N$, Wu Z: PCR-based identification of zoonotic isolates of Blastocystis from mammals and birds. Microbiology 2004, 150:1147-1151.

14. Özyurta M, Kurtb Ö, Mølbakc K, Nielsend HV, Haznedaroglua T, Stensvold CR: Molecular epidemiology of Blastocystis infections in Turkey. Parasitol Int 2008, 57:300-306.

15. Tan TC, Suresh KG: Predominance of amoeboid forms of Blastocystis hominis in isolates from symptomatic patients. Parasitol Res 2006, 98:189-193.

16. Tan TC, Suresh KG: Amoeboid form of Blastocystis hominis-a detailed ultrastructural insight. Parasitol Res 2006, 99:737-742.

17. Stenzel DJ, Boreham PFL, McDougall R: Ultrastructure of Blastocystis hominis in human stool samples. Int J Parasitol 1991, 21:807-812.

18. Lanuza M, Carbajal J, Villar J, Borrás R: Description of an improved method for Blastocystis hominis culture and axenization. Parasitol Res 1997, 83:60-63.

19. Sio SWS, Puthia MK, Lee ASY, Lu J, Tan KSW: Protease activity of Blastocystis hominis. Parasitol Res 2006, 99:126-130

20. Abdel-Hameed DM, Hassanin OM: Proteaese activity of Blastocystis hominis subtype3 in symptomatic and asymptomatic patients. Parasitol Res 2011, 109:321-327.

21. Puthia MK, Lu J, Tan KSW: Blastocystis ratti contains cysteine proteases that mediate interleukin-8 response from human intestinal epithelial cells in an NF-kB-dependent manner. Eukaryot Cell 2008, 7:435-443.

22. Puthia MK, Vaithilingam A, Lu J, Tan KSW: Degradation of human secretory immunoglobulin a by Blastocystis. Parasitol Res 2005, 97:386-389. 
23. Mirza $\mathrm{H}$, Tan KSW: Blastocystis exhibits inter- and intra-subtype variation in cystiene protease. Parasitol Res 2009, 104:355-361.

24. Suresh K, Ng GC, Ho LC, Yap EH, Singh M: Differentiation of the various stages of Blastocystis hominis by acridine orange staining. Int J Parasitol 1994, 24:605-606.

25. Que X, Reed SL: Cysteine proteinases and the pathogenesis of amebiasis. Clin Microbiol Rev 2000, 13:196-206.

26. Yadav M, Dubey ML, Gupta I, Bhatti G, Malla N: Cysteine proteinase 30 in clinical isolates of $T$. vaginalis from symptomatic and asymptomatic infected women. Exp Parasitol 2007, 116:399-406.

27. Khan NA, Jarroll EL, Panjwani N, Cao Z, Paget TA: Proteases as markers for differentiation of pathogenic and nonpathogenic species of acanthamoeba. J Clin Microbiol 2000, 38:2858-2861.

28. Wawrzyniak I, Texier C, Poirier P, Viscogliosi E, Tan KSW, Delbac F, Alaoui HE: Characterization of two cysteine proteases secreted by Blastocystis ST7, a human intestinal parasite. Parasitol Int 2012, 61:437-442.

29. Barrios-Ceballos MP, Martínez-Gallardo NA, Anaya-Velázquez F, Mirelman D, Padilla-Vaca F: A novel protease from Entamoeba histolytica homologous to members of the family S28 of serine proteases. Exp Parasitol 2005, 110:270-275.

30. Doyle PS, Zhou YM, Hsieh I, Greenbaum CD, McKerrow JH, Engel JC: The trypanosoma cruzi protease cruzain mediates immune evasion. PLoS Pathog 2011, 7:1-11.

31. Sissons J, Alsam S, Goldsworthy G, Lightfoot M, Jarroll EL, Khan NA: Identification and properties of proteases from an Acanthamoeba isolate capable of producing granulomatous encephalitis. BMC Microbiol 2006, 6:42-50.

32. Singh M, Suresh $K$, Ho LC, Ng GC, Yap EH: Elucidation of the life cycle of the intestinal protozoan Blastocystis hominis. Parasitol Res 1995, 81:446-450.

33. He N, Zhang $\mathrm{Y}$, Hong M, Cong M: Morphological and ultrastructural observation of Blastocystis hominis. Zhongguo Ji Sheng Chong Xue Yu Ji Sheng Chong Bing Za Zhi 2001, 19:169-172.

34. Zhang X, Zhang S, Qiao J, Wu X, Zhao L, Liu Y, Fan X: Ultrastructural insights into morphology and reproductive mode of Blastocystis hominis. Parasitol Res 2012, 110:1165-1172.

35. Scanlan PD: Blastocystis: past pitfalls and future perspectives. Trends Parasitol 2012, 28:327-334.

36. Carvalho TB, David EB, Coradi ST, Guimarães S: Protease activity in extracellular products secreted in vitro by trophozoites of Giardia duodenalis. Parasitol Res 2008, 104:185-190.

37. Tan TC, Suresh KG, Smith HV: Phenotypic and genotypic characterisation of Blastocystis hominis isolates implicates subtype 3 as a subtype with pathogenic potential. Parasitol Res 2008, 104:85-93.

38. Schulte W, Scholze H: Action of the major protease from Entamoeba histolytica on proteins of the extracellular matrix. J Protozool 1989, 36:538-543.

39. Samudi C, Kumar S, Anitab ZB, Kuppusamy UR: Infections of Blastocystis hominis and microsporidia in cancer patients: are they opportunistic? Trans R Soc Trop Med Hyg 2012, 106:267-269.

doi:10.1186/1756-3305-6-295

Cite this article as: Rajamanikam and Govind: Amoebic forms of Blastocystis spp. - evidence for a pathogenic role. Parasites \& Vectors 2013 6:295

\section{Submit your next manuscript to BioMed Central and take full advantage of:}

- Convenient online submission

- Thorough peer review

- No space constraints or color figure charges

- Immediate publication on acceptance

- Inclusion in PubMed, CAS, Scopus and Google Scholar

- Research which is freely available for redistribution

Submit your manuscript at www.biomedcentral.com/submit 